\title{
RESEARCH TO INDUSTRY AND INDUSTRY TO RESEARCH
}

\section{REMOVING POLLUTANTS, HEAVY METALS AND PFAS FROM LEACHATE USING MAG- NETIC FORCE}

Leachate is a consequence of landfill processes and operations, which, if not properly managed, monitored and treated, can be harmful to the surrounding environment. Landfill leachates consist of soluble organic and inorganic compounds as well as suspended particles. Depending on weather leachate flow can increase (during rainy season) or decrease (during dry/summer season).

The composition of waste changes dramatically over the life of the landfill due to chemical degradation and biological decay of organic matter present.

In general, landfill leachate pollutants can be categorized into four groups as dissolved organic matter, inorganic macro components, heavy metals, and organic compounds.

The $\mathrm{pH}$ of the leachate does not vary much and is often considered as alkaline as the $\mathrm{pH}$ is 7.0 to 8.5.

The current paradigm for wastewater and leachate treatment technologies are based on one or a combination of the following solutions:

\author{
Dissolved Air Flotation (DAF) \\ - Electro-coagulation; \\ - Aerated lagoon; \\ - Filtration; \\ - Biological treatment.
}

Some of the challenges a landfill owner is facing when choosing a well-suited technology is finding a solution that fits the existing space and constructions, the complexity of the treatment process, the operating expenses and how to handle the sludge from the treatment system in terms of both large volumes and the degree of dry matter content.

\section{Magnetic particle separation: a new hybrid system}

The treatment system developed by Mivanor AS is called MivaMag ${ }^{\mathrm{TM}}$, a combination of chemical and mechanical wastewater treatment (Figure 1).

\section{Chemical wastewater treatment}

Chemical treatment used in combination with magnetic particle separation is often based on coagulation and flocculation.

Suspended particles vary in source, charge, particle size,

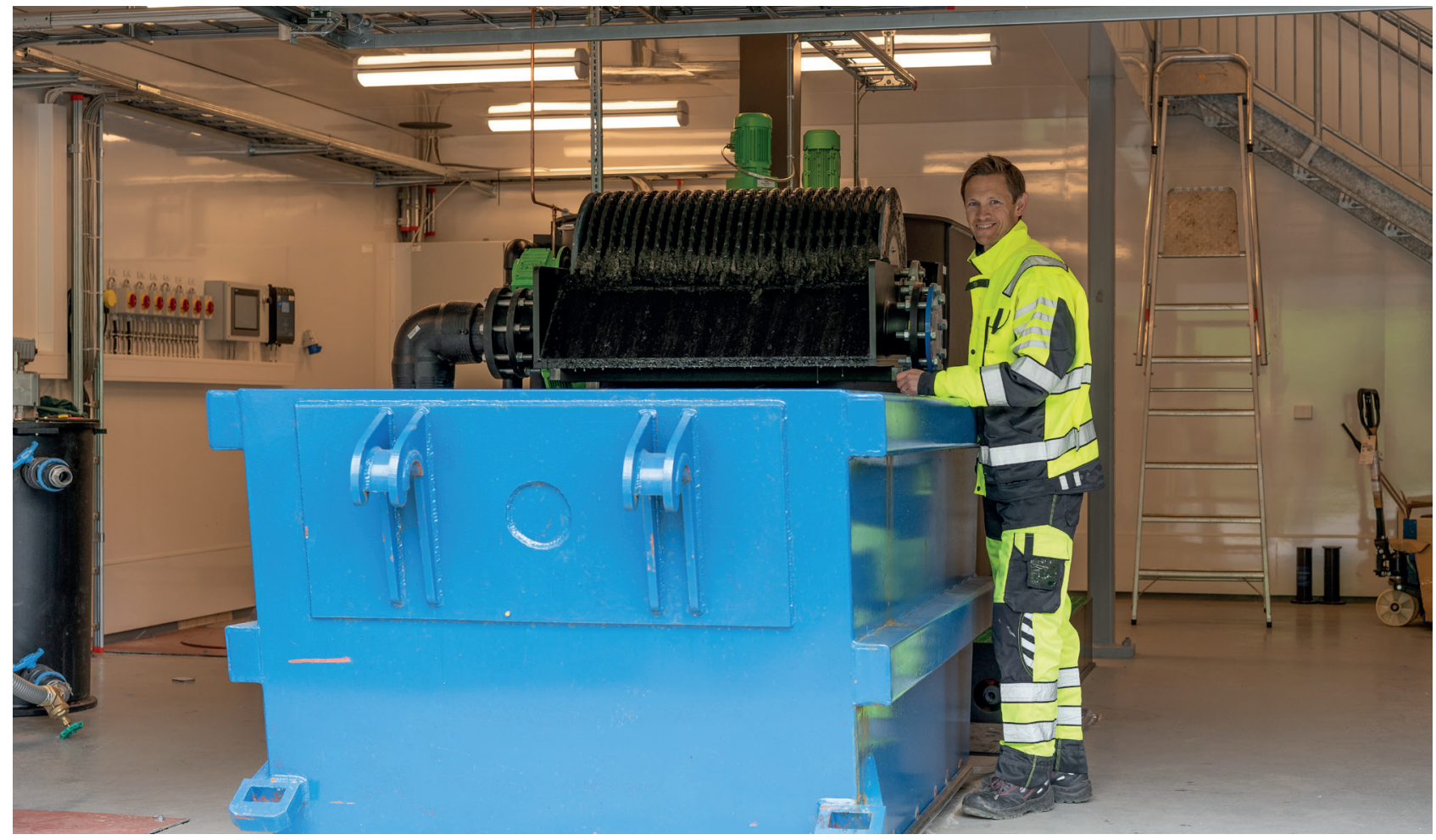

FIGURE 1: The tratment system "MivaMag ${ }^{\mathrm{TM}}$ ".

Detritus / Volume 09 - 2020 / pages III-V https://doi.org/10.31025/2611-4135/2020.13936 (C) 2020 Cisa Publisher 
shape, and density. Correct application of coagulation and flocculation depends upon these factors. Suspended solids in water are the main target and have a negative charge. Since they have the same type of surface charge, they repel each other when they come close together. Therefore, suspended solids will remain in suspension and will not clump together and settle out of the water, unless proper coagulation and flocculation is used.

Coagulant chemicals with positive charges are added to the water to neutralize the negative charges on non-settleable solids (such as clay and color-producing organic substances).

Inorganic coagulants such as aluminum and iron salts are the most commonly used.

Once the charge is neutralized, the small suspended particles can stick together and form so called "micro-floc".

Flocculation increases the particle size from submicroscopic micro-floc to visible suspended particles. Micro-floc particles collide, causing them to bond to produce larger, visible macro-flocs.

The main mechanism of building macro-flocs is based on bridge bonding using organic polymers.

\section{Magnetic Particle separation}

Untreated water is pumped into the system, and through mixers where a coagulant and flocculant agent are added. Further the water is led into basins where an adjusted circulation ensures an optimal capture and flocculation.

During flocculation an iron-based powder is added to the process. The powder consists mainly of $\mathrm{Fe}_{3} \mathrm{O}_{4}$ and it is called magnetite. Magnetite is a rock mineral and has a ferrimagnetic character.

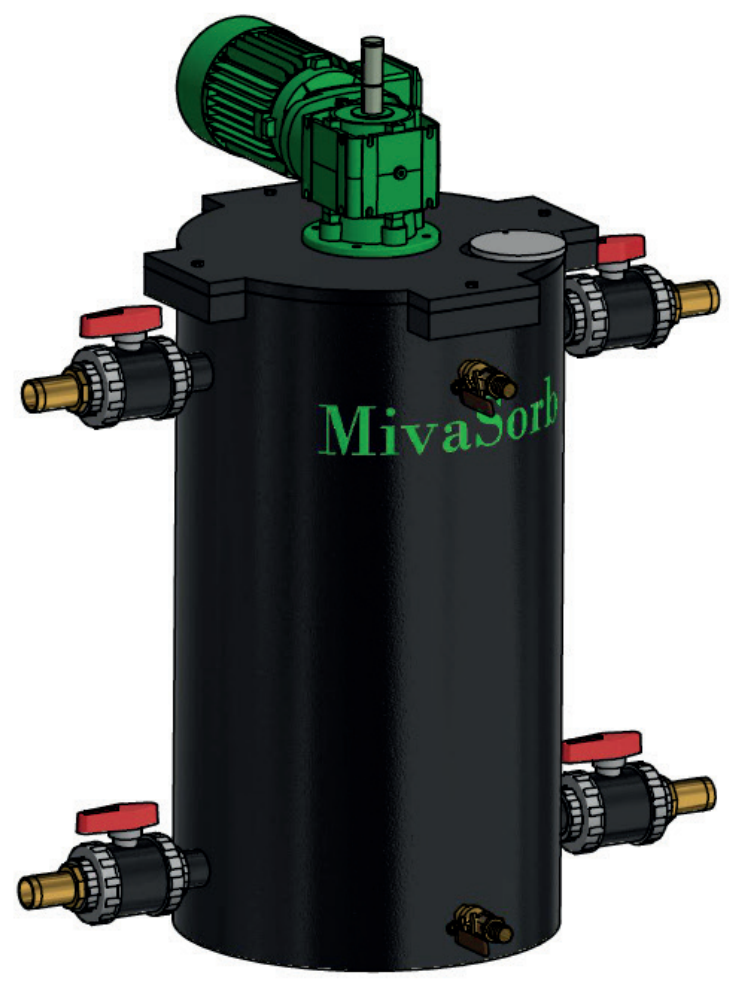

Adding magnetite to the treatment process enables the treatment system to extract the pollutants using magnets as the floc mass floats up to the surface of the basin, and is separated and pressured through a rotating, magnetic drum.

The pollutants are removed from the leachate at the same time as the sludge is dewatered. Dewatering happens due to magnetic forces where small amounts of water the pressed out of each floc.

The technology does not require use of a filter or an extra de-watering unit, something that makes it compact and cost-effective.

\section{Treatment results}

Each treatment unit has a capacity of 40 cubic meters of water per hour.

The results recorded from after treating leachate so far show that the magnetic particle separation reduces:

- $50-70 \%$ of the organic pollutants

- $25-95 \%$ of the of heavy metals

- $80-90 \%$ of the phosphorus

- $70-90 \%$ of the suspended particles

The sludge has a dry matter content of $30 \%$ to $40 \%$. The sludge is therefore easy to collect and transport for deposit.

\section{Expanding the leachate treatment to include pfas removal}

The treatment process is also possible to expand as Mivanor has developed a demonstrative treatment module for removing PFAS from leachate and wastewater (Figure 2). This research is on-going and Mivanor is exploring the treatment results from a couple of sites dealing with PFAS in water.

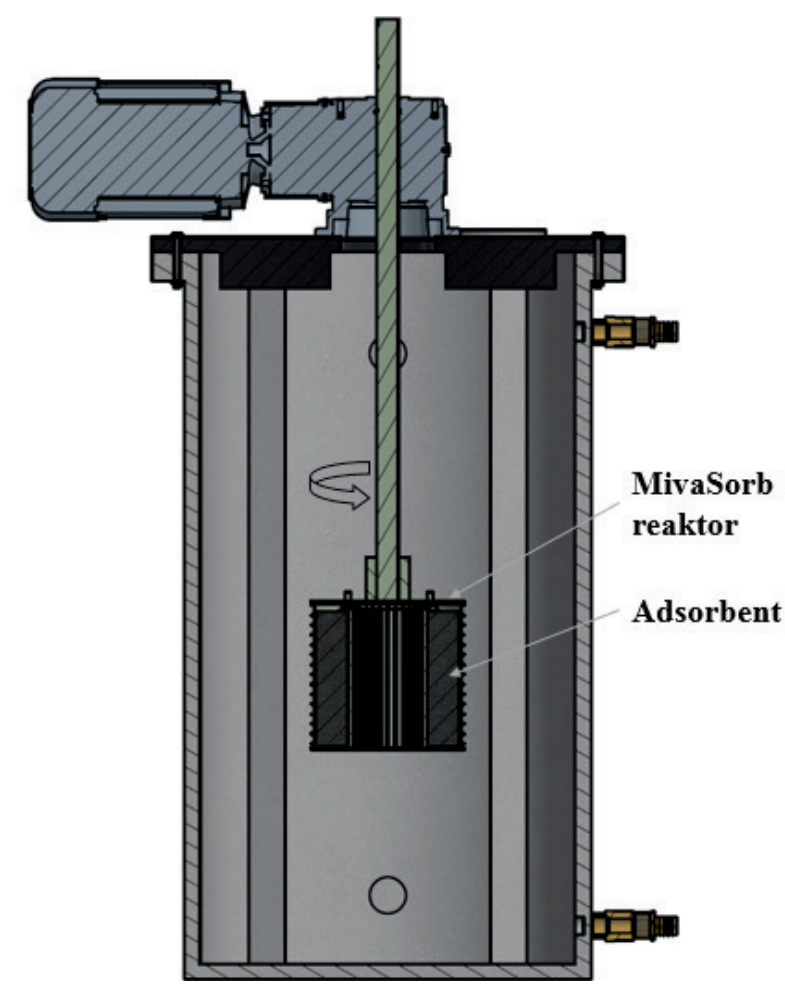

FIGURE 2: Demonstrative treatment module for the removal of PFAS. 
Per- and polyfluoroalkyl substances (PFAS) are a group of man-made chemicals that includes PFOA, PFOS and many other chemicals.

PFAS are used in many ordinary consumer goods, as well as industrial products because of the ability to make an object water and oil repellent. Some examples are frying pans, paints, outdoor clothing and also firefighting foam.

These chemicals are persistent in the environment and in the human body, meaning they don't break down and they can accumulate over time - leading to several negative health effects like cancer (for PFOA), thyroid hormone disruption (for PFOS) and general effects on the immune system.

For these reasons, PFAS has risen to be of great importance for the EU and in December 2019 EU launched a strategy for acting and minimize the exposure of PFAS in the environment and to human beings ${ }^{(1)}$.

Most landfill leachates that Mivanor has tested contain about 200-1000 ppt of PFAS mainly PFOS and PFOA.

To enable landfill owners to preserve masses containing PFAS in secured landfill areas and ensure that the leachate does not contaminate the surrounding nature with PFAS, Mivanor has developed a treatment solution that can be added to the magnetic particle separation process as a polishing step.

The product is called MivaSorb, and is designed to remove PFAS from polluted waste- or groundwater through adsorption in a rotating packed bed. MivaSorb consists of a cylindrical rotary container inside a vessel.

Due to rotary movement of the reactor, adsorption medium will enter the reactor from the bottom of the MivaSo$\mathrm{rb}$ vessel and water is circulated through the reactor and adsorption material. In this way the pollutants encounter the adsorbent many times over. Besides this, loading and re-loading of the reactor with adsorption medium can be automated.

The MivaSorb treatment method enable the treatment to be quite flexible in terms of the possibility to combine of several units and increase the flow capacity. The treatment system is designed so that the loading and reloading is automated, and there is no backwashing required.

Typical treatment results for the PFAS removal is:

- $90-99 \%$ reduction of PFAS compounds

- $90 \%$ reduction of other ions in water

Robert Tarasz *, Pernille Devold Kolsing

Mivanor, Postboks 6094, 8031 Bodø, Norway

www.mivanor.no

*email: robert@mivanor.no

Mivanor is a total supplier of treatment solutions for industrial wastewater. In order to ensure that our delivery will fulfil the customer's wishes and requirements, we supply each individual customer with a solution based on small-scale experiments with the relevant water and a carefully considered design.

This provides customers with a good estimate of the treatment results and operation costs prior to a system being ordered. Mivanor is ISO-certified in accordance with quality, health, safety and environment standards.

Today, the company has delivered 16 MivaMag ${ }^{\text {TM }}$ treatment plants.

(1) https://www.documentcloud.org/documents/6586418-EU-Strategy-for-PFASs-FINAL-VERSION-December-19.html 